\title{
Suppression of ССТЗ inhibits the proliferation and migration in breast cancer cells
}

\section{Gang Xu ( $\nabla$ xugang1981@126.com )}

Affiliated cancer hospital of Zhengzhou University https://orcid.org/0000-0002-2439-2075

\section{Shanshan Bu}

Zhengzhou University

\section{Xiushen Wang}

Zhengzhou University

He Zhang

Zhengzhou Universiy

Hong Ge

Zhengzhou University

\section{Primary research}

Keywords: ССТ3, breast cancer, proliferation, metastasis, cell cycle, apoptosis

Posted Date: April 15th, 2020

DOI: https://doi.org/10.21203/rs.3.rs-22398/v1

License: (c) (1) This work is licensed under a Creative Commons Attribution 4.0 International License.

Read Full License

Version of Record: A version of this preprint was published at Cancer Cell International on June 5th, 2020. See the published version at https://doi.org/10.1186/s12935-020-01314-8. 


\section{Abstract}

Background CCT3 is a subunit of chaperonin-containing TCP-1 (CCT), which folds many proteins involved in cancer development and plays an important role in many cancers. However, the role of CCT3 in breast cancer is still unclear. Methods СCT3 expression was knocked down by transfecting breast cancer cells with lentiviral shRNA. The proliferation of breast cancer cells (HCC1937 and MDA-MB-231) was detected by Celigo image cytometry and MTT assay, the migration of the cells was measured by Transwell analysis, cell cycle distribution and apoptosis was detected by flow cytometry, and changes in signal transduction proteins were detected by western blot analysis. Results The expression of CCT3 was significantly suppressed by transduction with lentiviral shRNA; CCT3 knockdown significantly reduced the proliferation and metastasis ability of breast cancer cells (HCC 1937 and MDA-MB-231), increased the proportion of cells in S phase, and decreased the proportion of cells in $\mathrm{G} 1$ phase compared to those in shControl cells. There was no significant change in the number of cells in the G2/M phase. Apoptosis analysis showed that knockdown of ССТ3 induced apoptosis in breast cancer cells. Western blot analysis showed that the expression of many signal transduction proteins was changed after suppression of CCT3. Conclusion CCT3 is closely related to the proliferation and migration of breast cancer and may be a novel therapeutic target.

\section{Background}

Breast cancer is a common malignant tumour in women. At present, the incidence rate of breast cancer is $24.2 \%$ worldwide. The mortality rate is also the highest among malignant tumours, accounting for approximately $15 \%$ of cancer-related deaths in women ${ }^{[1]}$. At present, the treatment of breast cancer mainly includes neoadjuvant therapy, surgery, chemotherapy, radiotherapy, targeted therapy and endocrine therapy ${ }^{[2]}$. The application of a comprehensive treatment mode improves the prognosis of breast cancer and prolongs the survival time of patients, but the overall effect is still unsatisfactory, especially for patients with stage IV metastasis, for whom the median total survival time is only 23 years ${ }^{[3]}$. Therefore, identification of a novel therapeutic target to treat breast cancer is an urgent need.

Chaperonins are molecules that assist in the folding of newly synthesized and stress-denatured polypeptide chains and are divided into two groups, group I and group II. Heat shock protein 60 (HSP60) or GroEL in bacteria belongs to group I, and chaperonin-containing TCP-1 (CCT or TRiC) belongs to group II. CCT is a large complex composed of two stacked rings, back-to-back, consisting of eight distinct subunits (CCT1-CCT8) ${ }^{[4-6]}$. In cancer cells, CCT folds proteins related to carcinogenesis, such as kirsten rat sarcoma viral oncogene (KRAS), Signal transducers and activators of transcription 3 (STAT3), and p53. ССТ3 is an important subunit of CCT and is widely studied in different cancers. The mRNA and protein expression of CCT3 in hepatocellular carcinoma (HCC) tissues are higher than those in non-HCC tissues, and СCT3 plays an important role in the tumorigenesis and progression of HCC and has prognostic value in $\mathrm{HCC}{ }^{[7,8]}$. Further study showed that CCT3 is a novel regulator of spindle integrity and is required for proper kinetochore-microtubule attachment during mitosis ${ }^{[9]}$. In gastric cancer, a higher 
level of CCT3 expression was detected in tumour tissues than in non-cancerous epithelial tissues.

Knockdown of ССТ3 inhibited the proliferation and survival of gastric cancer cells ${ }^{[10]}$. In papillary thyroid carcinoma, knockdown of CCT3 decreased the proliferation and cell cycle progression and induced the apoptosis of $\mathrm{K} 1$ cells ${ }^{[11]}$. In multiple myeloma, СCT3 was also a significant indicator of poor prognosis, and CCT3 expression was associated with the JAK-STAT3 pathway, Hippo signalling pathway, and WNT signalling pathway ${ }^{[12]}$.

Genomic analysis of the Cancer Genome Atlas, which contains data for 971 cases of breast carcinoma with sequencing and copy number analysis, showed that $51 \%$ of cases have alterations in at least one CCT subunit and that the highest alteration rate occurred in CCT3 (31\%) ${ }^{[13]}$. However, whether CCT3 regulates the development of breast cancer is still unknown.

In the present study, we found that knockdown of ССТ3 inhibits the proliferation and metastasis of breast cancer cells and that the mechanism is probably related to regulation of the cell cycle, apoptosis and several signal transduction pathways.

\section{Materials And Methods}

\section{Cells and materials}

HCC1937 and MDA-MB-231 cell lines were purchased from the Cell Bank of the Chinese Academy of Science (Shanghai, China); 3-(4,5-dimethylthiazol-2-yl)-2,5-diphenyltetrazolium bromide (MTT) was purchased from Genview (Campbellfield, VIC, Australia); SYBR Master Mixture was purchased from Takara (Shimogyo-ku, Kyoto, Japan); antibodies against CCT3, CDH1, Slug, Snail, VIM, mTOR, ERK1/2, pERK1/2, p-AKT1, P38, p-P38, NFKB-65, p-NFKB-65, $\beta$-catenin and p- $\beta$-catenin were purchased from Cell Signaling Technology (Danvers, MA, USA); and antibodies against CDH2, MMP2, FN1, MYC, p-mTOR and AKT1 were purchased from Abcam (Cambridge, MA, USA). GAPDH antibody was purchased from Santa Cruz Biotechnology, Inc. (Dallas, TX, USA).

\section{Cell culture}

HCC1937 and MDA-MB-231 cell lines were cultured in RPMI 1640 medium(Gibco; Gaithersburg, MD, United States) supplemented with $10 \%$ foetal bovine serum. The cells were maintained at $37^{\circ} \mathrm{C}$ in a $5 \%$ $\mathrm{CO}_{2}$ humidified incubator.

\section{Lentiviral vector construction and transduction}

For the construction of shRNA expression plasmids, shCCT3 was designed based on the target sequence 5'-CAAGTCCATGATCGAAATT-3'. Then, the single strand DNA oligo containing the interference sequence was synthesized, and the double strand DNA was produced by annealing. Then, the two ends of the oligo were directly linked to the lentiviral vector after enzyme digestion. The ligated products were transferred into the prepared Escherichia coli cells. Then, the positive recombinants were identified by PCR and 
sequenced for verification and plasmid extraction. Lentiviral vector DNA and packaging vectors were transfected into 293T cells. The empty GV115 lentiviral vector was used as the shRNA control (shCtrl). After $48 \mathrm{~h}$ of culture, supernatants containing lentiviruses, including shCCT3 and shCtrl, were harvested and purified. Lentiviral transduction was performed on cells at $80 \%$ confluency, with a multiplicity of infection (MOI) of 10. Seventy-two hours after infection, the cells were used for downstream assays or transplantation.

\section{QRT-PCR analysis}

Total RNA was isolated by the TRIzol method. The cDNA reverse-transcribed from $250 \mathrm{ng}$ of total RNA was amplified using the following primer sets: CCT3: forward, 5'-TCA GTC GGT GGT CAT CTT TGG-3', reverse, 5'-CCT CCA GGT ATC TTT TCC ACT CT-3'; and GAPDH: forward, 5'-TGA CTT CAA CAG CGA CAC CCA-3', reverse, 5'-CAC CCT GTT GCT GTA GCC AAA-3'. Real-time PCR using the SYBR Green PCR Master Mix kit was performed with an ABI Prisma 7000 Sequence Detection System (Applied Biosystems, Foster City, CA, USA) following the manufacturer's instructions. Data were normalized to the respective GAPDH values. The value of cells infected with shControl(shCtrl) was set to $100 \%$ in each run.

\section{Celigo image cytometry assay}

The cells were trypsinized in the logarithmic growth phase, resuspended in medium, seeded into 96-well plates at $2 \times 10^{3}$ cells $(100 \mu \mathrm{l})$ per well, and incubated overnight. A Celigo Image Cytometer (Nexcelom Bioscience, Lawrence, MA, USA) was used to evaluate the number of cells by scanning for green fluorescence daily for 5 consecutive days at room temperature. The cell proliferation curve was plotted according to the number of cells with green fluorescence.

\section{MTT assay}

HCC1937 and MDA-MB-231 cells infected with shCCT3 or shCtrl were seeded into 96-well plates at $1.5 \times 10^{3}$ cells and $2 \times 10^{3}$ cells per well, respectively, and incubated overnight. The cells were cultured for 5 days at $37^{\circ} \mathrm{C}$. MTT assays were carried out at different time points: $24 \mathrm{~h}, 48 \mathrm{~h}, 72 \mathrm{~h}, 96 \mathrm{~h}$ and $120 \mathrm{~h}$. Then, $20 \mu \mathrm{l}$ MTT solution $\left(5 \mathrm{mg} / \mathrm{ml}\right.$ ) was added to each well and incubated for an additional $4 \mathrm{~h}$ at $37^{\circ} \mathrm{C}$. Then, the MTT solution was aspirated, and $100 \mu \mathrm{LMSO}$ was added to dissolve the formazan crystals. The number of cells was counted using a microplate reader at a wavelength of $490 \mathrm{~nm}$.

\section{Transwell migration assay}

HCC1937 and MDA-MB-231 cells infected with shCCT3 or the shCtrl were seeded on Transwell inserts $\left(6.5 \mathrm{~mm}, 8 \mu \mathrm{m}\right.$ pores) coated with or without Matrigel in 24 -well plates at $60 \times 10^{3}$ cells and $80 \times 10^{3}$ cells per well, respectively, and then placed in the incubator to culture for $24 \mathrm{~h}$. Cells on the upper side of the insert were scraped away, and then the inserts were fixed and stained. Invaded cells were counted under an inverted microscope. 
The cells were seeded in 6-well plates for apoptosis analysis or a 6-cm dish for cell cycle analysis. The cells were trypsinized at 70-80\% confluency, suspended and washed with D-Hanks solution. For apoptosis analysis, cells were resuspended and stained with annexin V-APC. For cell cycle analysis, cells were fixed with $75 \% \mathrm{EtOH}$ at $-20^{\circ} \mathrm{C}$ for at least $2 \mathrm{~h}$ and then harvested and stained with $\mathrm{PI}(10 \mathrm{ng} / \mathrm{ml})$ and RNase (10 $\mathrm{ng} / \mathrm{ml})$. Then, the cells were submitted to flow cytometry analysis.

\section{Western blot analysis}

The proteins were separated by $10 \%$ sodium dodecyl sulfate-polyacrylamide gel electrophoresis (SDSPAGE) (Beyotime, Shanghai, China) and then transferred to polyvinylidene fluoride membranes (Millipore, Billerica, MA, USA), which were blocked for $2 \mathrm{~h}$ with $5 \%$ nonfat milk. The membranes were incubated with primary antibodies against CCT3 (1:300), CDH1 (1:200), CDH2 (1:1000), Slug (1:1000), MMP2 (1:200), FN1 (1:300), Snail (1:1000), MYC (1:500), VIM (1:1000), ERK1/2 (1:1000), p-ERK1/2 (1:1000), AKT1 (1:1000), p-AKT (1:2000), $\beta$-catenin (1:1000), p- $\beta$-catenin (1:1000), mTOR (1:1000), p-mTOR (1:2000), NFKB-65 (1:1000), p-NFKB-65 (1:1000), P38 (1:1000), p-P38 (1:1000), and GAPDH (1:2000) overnight at $4^{\circ} \mathrm{C}$. Next, the membranes were incubated with horseradish peroxidase (HRP)-conjugated secondary antibodies $(1: 2000)$ for $1 \mathrm{~h}$ at room temperature. The blots were visualized using the Super Signal West Femto kit (Pierce Biotechnology, Rockford, IL, USA).

\section{Statistical analysis}

All statistical analyses were performed using SPSS 19.0 software (SPSS, Chicago, IL, USA). Data are represented as the mean \pm standard deviation. All experiments were performed in triplicate. Student's ttest or one-way analysis of variance (ANOVA) was used for statistical analysis. For ANOVA, when a significant difference was apparent, Dunnett's post hoc test was used to compare the means of multiple experimental groups. Differences with $p<0.05$ were considered statistically significant.

\section{Results}

\section{Knockdown of ССТ3 expression inhibited breast cancer cell proliferation}

Lentivirus expressing short hairpin RNA targeting CCT3 mRNA (shCCT3) was used to infect the breast cancer cell lines HCC1937 and MDA-MB-231. As shown in Fig 1A, the cell transduction rate was above $80 \%$. The silencing effect of CCT3 in HCC1937 and MDA-MB-231 cells was measured by qRT-PCR and western blotting. The expression of CCT3 mRNA decreased by $84.4 \%$ and $92.1 \%$ after transduction with lentivirus shCCT3 in the HCC1937 and MDA-MB-231 cells compared with that in the shCtrl cells, respectively (Fig 1B). ССT3 protein expression also decreased after transduction with shCCT3, which was confirmed by western blot analysis (Fig 1C). Celigo image cytometry assay and MTT assay showed that the proliferation of the breast cancer cells was inhibited significantly in shCCT3 cells compared to shCtrl cells (Fig 2, Fig 3). 
The migration ability of HCC1937 and MDA-MB-231 breast cells was analysed by Transwell assays with or without Matrigel. In Transwell assays without Matrigel, the migration of the shCCT3 group was 16\% and $21 \%$ of that in the shCtrl group in HCC1937 and MDA-MB-231 cells, respectively. In Transwell assays with Matrigel, the migration in the shCCT3 group was $45 \%$ and $43 \%$ of that in the shCtrl group in HCC1937 and MDA-MB-231 cells, respectively. As shown in Fig 4, after transduction with lentivirus shCСT3, the migration of the cells decreased significantly compared to that in the shCtrl group.

\section{Knockdown of СCT3 promoted apoptosis in breast cancer cells}

The annexin V-APC single staining method was used for apoptosis analysis. The percentage of apoptotic cells in the shCtrl group was $2.92 \%$ and $2.81 \%$ and increased to $11.08 \%$ and $6.32 \%$ in the shCCT3 group in HCC1937 cells and MDA-MB-231 cells, respectively. As shown in Fig 5, knockdown of CCT3 promoted apoptosis in the breast cancer cell lines HCC1937 and MDA-MB-231.

\section{Knockdown of ССТЗ regulated cell cycle distribution in breast cancer cells}

After transduction with shCtrl or shCСТ 3 for 5 days, the cell cycle distribution was measured by flow cytometry. The number of cells (HCC1937 and MDA-MB-231) in S phase increased, the number in G1 phase decreased, and there was no significant change in G2/M phase in the shCCT3 group compared with that in the shCtrl group (Fig 6).

\section{Knockdown of ССТ3 regulated cell cycle progression in breast cancer cells}

To explore possible signal transduction pathways affected by the ССТ3 gene, we detected several signal transduction proteins by western blot analysis in MDA-MB-231 cells. The results showed that MMP2, Snail, and VIM were upregulated and that CDH1, CDH2, Slug, FN1, MYC, ERK1/2, p-ERK1/2, AKT1, p-AKT1, $\beta$-catenin, $p$ - $\beta$-catenin, p-mTOR, NFKB-p65, p- NFKB-p65 and p-P38 were downregulated in MDA-MB-231 cells infected with shCCT3 compared to cells infected with shCtrl. There were no significant differences in mTOR and P38 between the 2 groups.

\section{Discussion}

Currently, the treatments for breast cancer include surgery, endocrine therapy, radiation therapy, chemotherapy, and targeted therapy. Among them, targeted therapy, such as trastuzumab, CDK4/6 inhibitors, and PI3K/Akt/mTOR inhibitors, significantly prolongs the survival time of patients with breast cancer ${ }^{[14-16]}$. However, the overall survival is not satisfactory, especially in patients with advanced breast cancer. Therefore, we wanted to search for a novel therapeutic target to improve the therapeutic effect and prolong the survival time.

ССТЗ is an important subunit of the molecular chaperone ССТ and is involved in the folding process of $7 \%$ of all cytosolic proteins, such as cytoskeletal proteins (tubulins, actins), cyclin E and Von HippelLindau $(\mathrm{VHL})^{[6,17,18]}$. These proteins determine the central role of CCT in the proliferation of cancer cells. 
The expression of ССТ3 in cancer tissue is higher than that in non-cancerous tissue, and knockdown or suppression of the expression of ССТ3 can inhibit the proliferation of cancer cells in many malignant carcinomas, such as hepatocellular carcinoma ${ }^{[9]}$, gastric carcinoma ${ }^{[10]}$, and papillary thyroid carcinoma ${ }^{[11]}$. In our study, we found that transduction with the lentiviral shRNA targeting CCT3 suppressed the mRNA and protein expression of CCT3 in the breast cancer cell lines HCC1937 and MDAMB-231. MTT assay and Celigo analysis showed that knockdown of ССТ3 inhibited the proliferation of breast cancer cells, which is consistent with reports in other tumours.

Rearrangement of actin filaments plays an important role in cancer cell migration or invasion ${ }^{[19]}$. P-21activating kinase PAK4 and gelsolin are actin regulators that are known to bind to $\mathrm{CCT}^{[20,21]}$. Therefore, inhibiting the expression of CCT should decrease the migration ability of cancer cells. Indeed, many reports have shown that knockdown of CCT inhibits the migration and invasion of some cancer cells ${ }^{[22-}$ 24]. In our study, we also found that knockdown of ССТ3 inhibited the migration of breast cancer cells through Transwell analysis.

The relationship between CCT and the cell cycle has been well reported ${ }^{[6,25]}$. Many cell cycle regulatory proteins are substrates of CCT, such as tubulin, Cdc20, and Cdh1. Tubulin synthesis increases around the G1/S transition, and a CCT-tubulin interaction has been observed in early S phase ${ }^{[26]}$. Cdc20 and Cdh1 are important at the transition from metaphase to anaphase ${ }^{[27]}$. Therefore, many reports have shown that suppression of CCT3 can induce S phase arrest. In this study, we found that knockdown of CCT3 increased the number of cells in S phase and decreased the number of cells in G1 phase, while the number of cells in G2/M phase was not significantly altered.

Apoptosis plays an important role in the carcinogenesis, development and treatment of breast cancer ${ }^{[28]}$. It has been reported that inhibition of ССТ3 can induce apoptosis ${ }^{[11]}$. We confirmed that knockdown of CCT3 can induce apoptosis in breast cancer with the annexin method in this study.

ССT3 is involved in STAT3 protein folding ${ }^{[29]}$, and many reports have confirmed that the effect of CCT3 is achieved via the JAK-STAT3 pathway. Therefore, we tried to explore other signalling pathways. We found that CCT3 can regulate a variety of signalling pathways in breast cancer cells, some of which play an important role in tumour development. The results showed that Snail, VIM and MMP2 were upregulated and that CDH1, CDH2, ERK1/2, p-ERK1/2, p-P38, FN1, AKT1, p-AKT1, MYC, NFkB-p65, p- NFkB-p65, pmTOR, $\beta$-catenin, $p$ - $\beta$-catenin and Slug were downregulated in MDA-MB-231 cells infected with shCCT3 compared to those infected with shCtrl. In future studies, a verification test will be carried out to confirm the role of these signal transduction pathways in breast cancer.

\section{Conclusions}

In our study, we found that knockdown of ССТ3 can inhibit the proliferation and metastasis of breast cancer cells, induce apoptosis and regulate the cell cycle. The effect of ССТ3 may be achieved through a variety of signalling pathways. ССТ3 may be a novel therapeutic target for breast cancer. 


\section{Abbreviations}

HSP 60: heat shock protein 60; CCT: chaperonin-containing TCP-1; KRAS: kirsten rat sarcoma viral oncogene; STAT3: signal transducers and activators of transcription 3; HCC: hepatocellular carcinoma; MTT: 3-(4,5-dimethylthiazol-2-yl)-2,5-diphenyltetrazolium bromide; MOI: multiplicity of infection; SDSPAGE: sodium dodecyl sulfate-polyacrylamide gel electrophoresis; ANOVA: one-way analysis of variance; VHL: Von Hippel-Lindau.

\section{Declarations}

\section{Authors' contributions}

XG designed the study, WX, BS and ZH performed the experiments. GH supervised the study, and provided technical assistance with experiments. $X G$ and $W X$ wrote the manuscript. All co-authors have reviewed this version of the manusucript. All authors read and approved the final manuscript.

\section{Funding}

This work was supported by the Henan Science and Technology projects (No.192102310102).

\section{Availability of data and meterials}

All data generated or analysed during this study are included in this published article.

\section{Competing interests}

The authors declare that they have no competing interests.

\section{Author details}

${ }^{1}$ Department of Radiation Oncology, Affiliated Cancer Hospital, Zhengzhou University. Henan province, China. ${ }^{2}$ Department of Pathology, Affiliated Cancer Hospital, Zhengzhou University. Henan province, China.

\section{References}

1. Freddie Bray J, Ferlay I, Soerjomataram RL, Siegel LA, Torre A. Jemal. Global cancer statistics 2018: GLOBOCAN estimates of incidence and mortality worldwide for 36 cancers in 185 countries. CA Cancer J Clin. 2018;68(6):394-424. doi:.

2. Nadia Harbeck. Frédérique Penault-Llorca 10.1038/s41572-019-0111-2 Nadia Harbeck. Frédérique Penault-Llorca. Cortes J, Gnan M, Houssami N, Poortmans P, Ruddy K, Janice Tsang, Fatima Cardoso. Breast cancer. Nat Rev Dis Primers. 2019;5(1):66. doi:. 
3. Cardoso F, Senkus E, Costa A, Papadopoulos E, Aapro M, André F, Harbeck N, Aguilar Lopez B, Barrios $\mathrm{CH}$, Bergh J, et al. 4th ESO-ESMO international consensus guidelines for advanced breast cancer (ABC 4)t. Ann Oncol. 2018; 1634-57. Doi:.

4. Stoldt V, Rademacher F, Kehren V, Ernst JF, Pearce DA, Sherman F. Review: the CCT eukaryotic chaperonin subunits of Saccharomyces cerevisiae and other yeasts. Yeast. 1996;12:523-9.

5. Valpuesta JM, Martin-Benito J, Gomez-Puertas P, Carrascosa JL, Willison KR. Structure and function of a protein folding machine: the eukaryotic cytosolic chaperonin CCT. FEBS Lett. 2002;529:11-6.

6. Karen I, Brackley J. Grantham. Activities of the chaperonin containing TCP-1 (CCT): implications for cell cycle progression and cytoskeletal organisation. Cell Stress Chaperones. 2009;14(1):23-31.

7. $10.1186 / s 12935-016-0332-3$

Qian E-Na, Han Shuang-Yin, Ding Song-Ze, Xun Lv. Expression and diagnostic value of CCT3 and IQGAP3 in hepatocellular carcinoma. Cancer Cell Int. 2016; 16:55. Doi:.

8. Hou J-Y, Wu H-Y, He R-Q, Lin P, Dang Y-W, Chen G. Clinical and prognostic value of chaperonin containing T-complex 1 subunit 3 in hepatocellular carcinoma: a study based on microarray and RNA-sequencing with 4272 cases. Pathol Res Pract. 2019;215(1):177-94.

9. Zhang Y, Wang Y, Wei Y, Wu J, Zhang P, Shen S, Saiyin H, Wumaier R, Yang X, Wang C, et al. Molecular chaperone CCT3 supports proper mitotic progression and cell proliferation in hepatocellular carcinoma cells. Cancer Lett. 2016;372(1):101-9.

10. Li LJ, Zhang LS, Han ZJ, He ZY, Chen H, Li YM. Chaperonin containing TCP-1 subunit 3 is critical for gastric cancer growth. Oncotarget. 2017;8(67):111470-81.

11. Shi XH, Cheng SS, Wang WX. Suppression of CCT3 inhibits malignant proliferation of human papillary thyroid carcinoma cell. Oncol Lett. 2018;15(6):9202-8.

12. Qian TT, Cui LZ, Liu Y, Cheng ZH, Quan L, Zeng TS, Huang WH, Dai YF, Chen JH, Liu L. High expression of chaperonin-containing TCP1 subunit 3 may induce dismal prognosis in multiple myeloma. Pharmacogenomics J. 2020. . doi:10.1038/s41397-019-0145-6.

13. Rania Bassiouni KN, Nemec A, Iketani O, Flores A, Showalter, Amr S, Khaled P, Vishnubhotla RW, Sprung C Jr, Kaittanis, Jesus M, Perez, et al. Chaperonin containing TCP-1 protein level in breast cancer cells predicts therapeutic application of a cytotoxic peptide. Clin Cancer Res. 2016;22(17):4366-79. doi:.

14. Waks AG, Winer EP. Breast cancer treatment: a review. JAMA. 2019;321(3):288-300. doi:.

15. Ribnikar D, Volovat SR, Cardoso F. Targeting CDK4/6 pathways and beyond in breast cancer. Breast. 2019;43:8-17. doi:.

16. Sharma VR, Gupta GK, Sharma AK, Batra N, Sharma DK, Joshi A, Anil K, Sharma. PI3K/Akt/mTOR intracellular pathway and breast cancer: factors, mechanism and regulation. Curr Pharm Des. 2017;23(11):1633-8. doi:.

17. 10.1073 /pnas. 90.20 .9422

Sternlicht H, Farr GW, Sternlicht ML, Driscoll JK, Willison KR, Yaffe MB. The t-complex polypeptide 1 complex is a chaperonin for tubulin and actin in vivo. Proc. Natl Acad. Sci. 1993;90, 9422-9426. doi:. 
18. Willison KR. The substrate specificity of eukaryotic cytosolic chaperonin CCT. Philos Trans R Soc Lond B Biol Sci. 2018;373(1749):20170192. doi:.

19. Olson MF, Sahai E. The actin cytoskeleton in cancer cell motility. Clin Exp Metastasis. 2009;26:27387.

20. Zhao M, Spiess M, Johansson HJ, Olofsson H, Hu J, Lehtio J, Stromblad S. Identification of the PAK4 interactome reveals PAK4 phosphorylation of N-WASP and promotion of Arp2/3-dependent actin polymerization. Oncotarget. 2017;8:77061-74.

21. Brackley KI, Grantham J. Interactions between the actin filament capping and severing protein gelsolin and the molecular chaperone CCT: evidence for nonclassical substrate interactions. Cell Stress Chaperones. 2011;16:173-9.

22. Cui X, Hu ZP, Li Z, Gao PJ, Zhu JY. Overexpression of chaperonin containing TCP1, subunit 3 predicts poor prognosis in hepatocellular carcinoma. World J Gastroenterol. 2015;21(28):8588-604. doi:.

23. Liu YJ, Chang YJ, Kuo YT, Liang PH. Targeting $\beta$-tubulin/CCT- $\beta$ complex induces apoptosis and suppresses migration and invasion of highly metastatic lung adenocarcinom. Carcinogenesis. 2019. doi:.

24. Yang X, Ren H, Shao Y, Sun Y, Zhang L, Li H, Zhang X, Yang X, Yu W, Fu J. Chaperonin-containing Tcomplex protein 1 subunit 8 promotes cell migration and invasion in human esophageal squamous cell carcinoma by regulating a-actin and $\beta$-tubulin expression. Int J Oncol. 2018;52(6):2021-30. doi:.

25. Grantham J, Brackley KI, Willison KR. Substantial CCT activity is required for cell cycle progression and cytoskeletal organization in mammalian cells. Exp Cell Res. 2006;312(12):2309-24. doi:.

26. Yokota S, Yanagi H, Yura T, Kubota H. Cytosolic chaperonin is upregulated during cell growth. Preferential expression and binding to tubulin at $G(1) / S$ transition through early S phase. J Biol Chem. 1999;274:37070-8.

27. Camasses A, Bogdanova A, Shevchenko A, Zachariae W. The CCT chaperonin promotes activation of the anaphase-promoting complex through the generation of functional Cdc20. 2003; Mol Cell. 12:87-100.

28. Kadam CY, Abhang SA. Apoptosis markers in breast cancer therapy. Adv Clin Chem. 2016;74:14393. doi:.

29. Kasembeli M, Lau WC, Roh SH, Echkols TK, Frydman J, Chiu W, Tweady DJ. Modulation of STAT3 folding and function by TRiC/CCT chaperonin. PLoS Biol. 2014;12(4):e1001844. doi:.

\section{Figures}


A

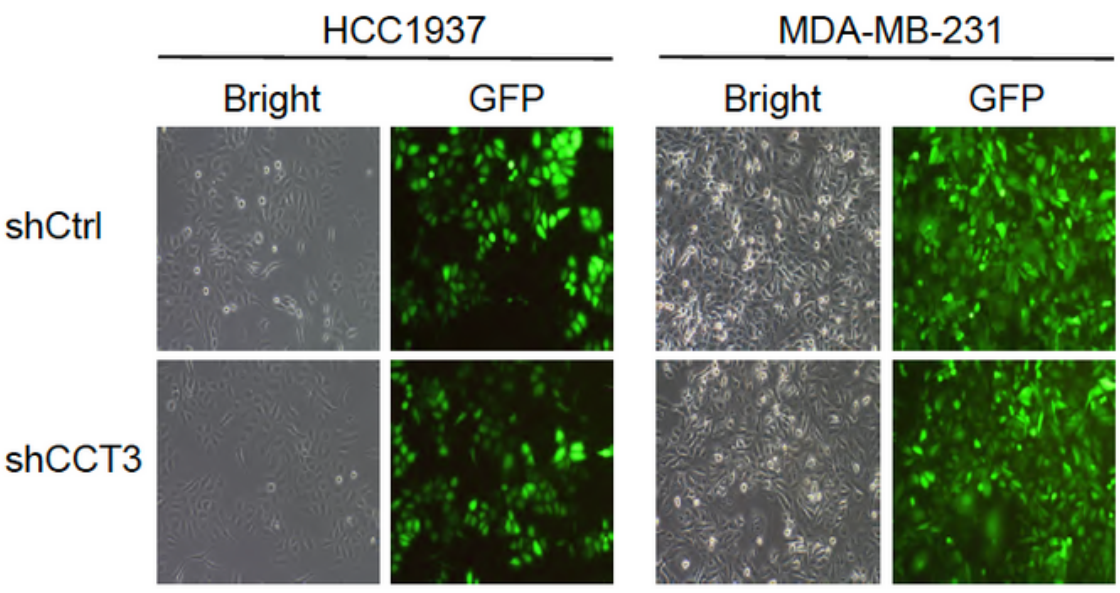

B

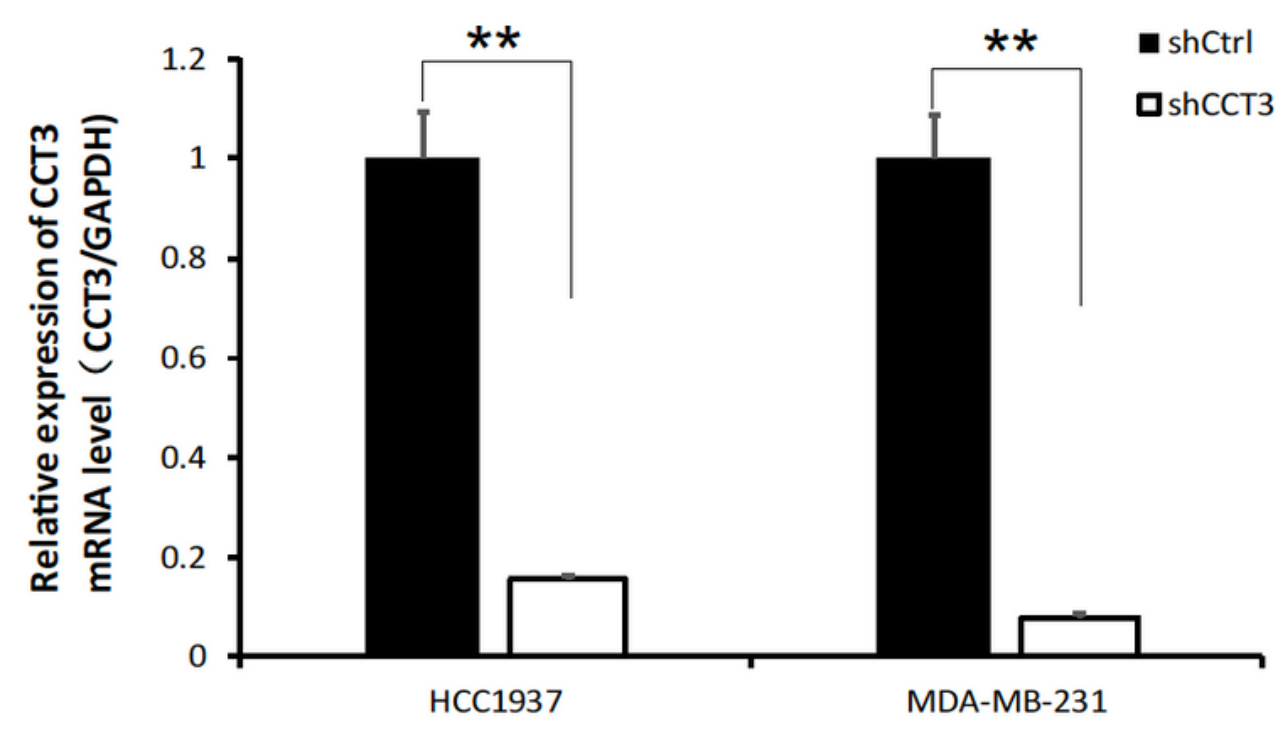

C

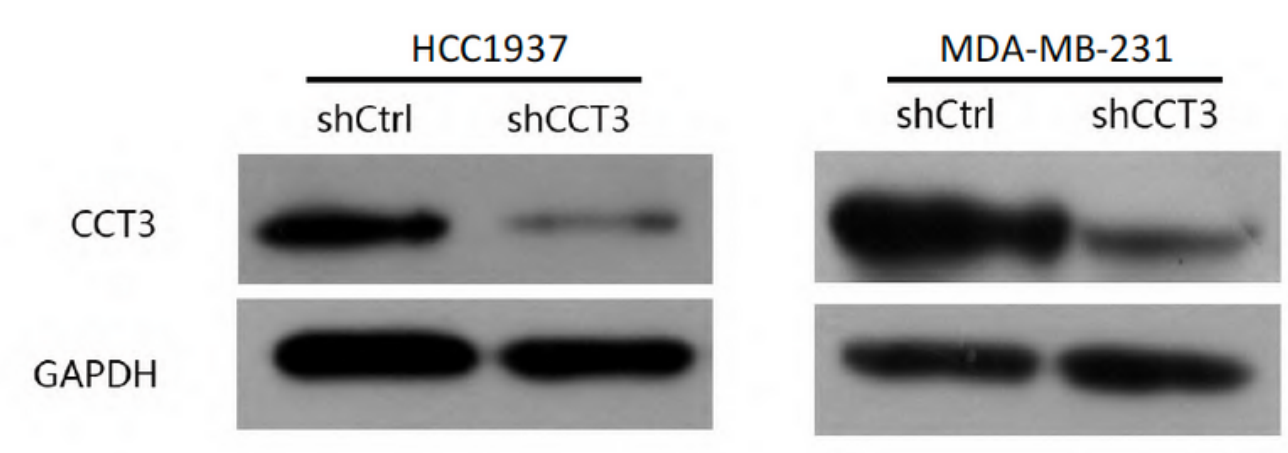

\section{Figure 1}

Knock of CСТ 3 in breast cancer cells. (A) Infection efficiency was determined at $72 \mathrm{~h}$ after infection of lentivirus shCCT3 or shCtrl in HCC1937 and MDA-MB-231 cells. Original magnification $\times 100$. (B) CCT3 mRNA in breast cancer cells was measured by realtime RT-PCR, and normalized to GAPDH. Data was presented as the mean \pm standard error. (C) CCT3 protein expression was analyzed by western blot analysis in HCC1937 and MDA-MB-231 infected with shCCT3 or shCtrl. ** $p<0.01$ shCCT3 vs shCtrl. 
A
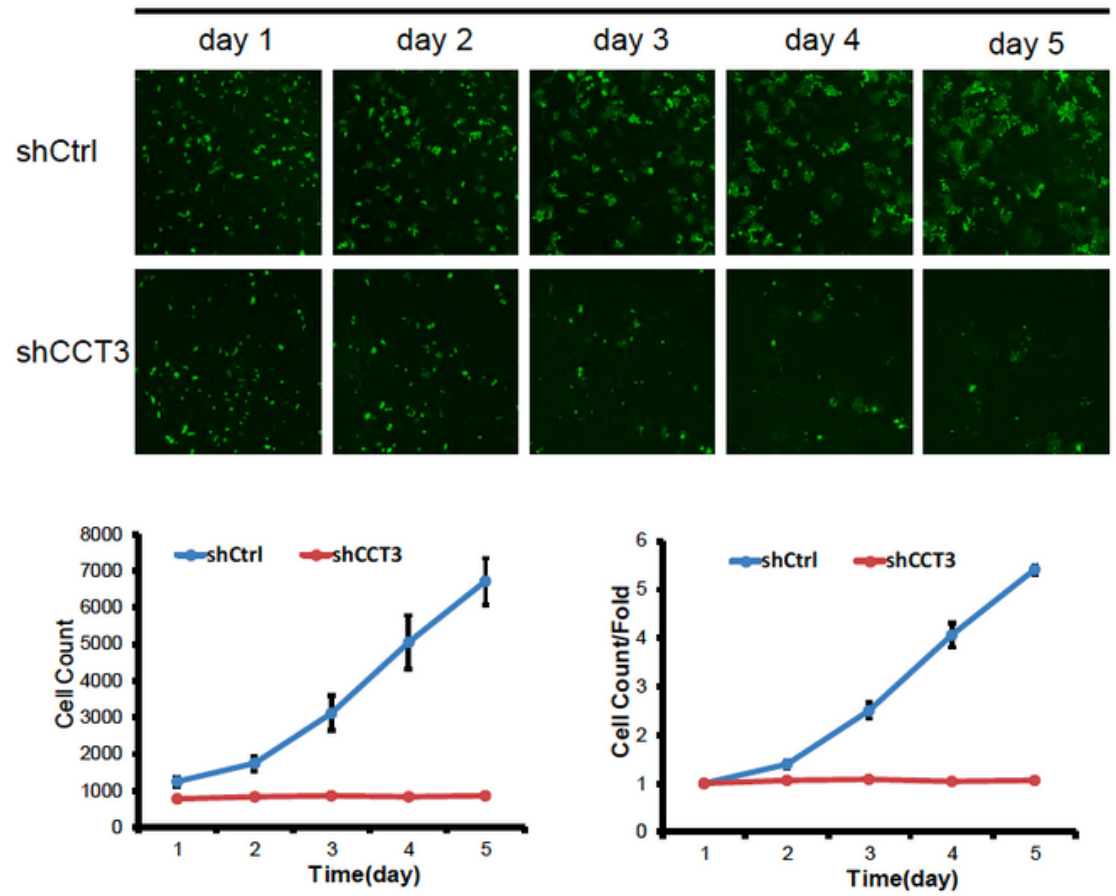

B
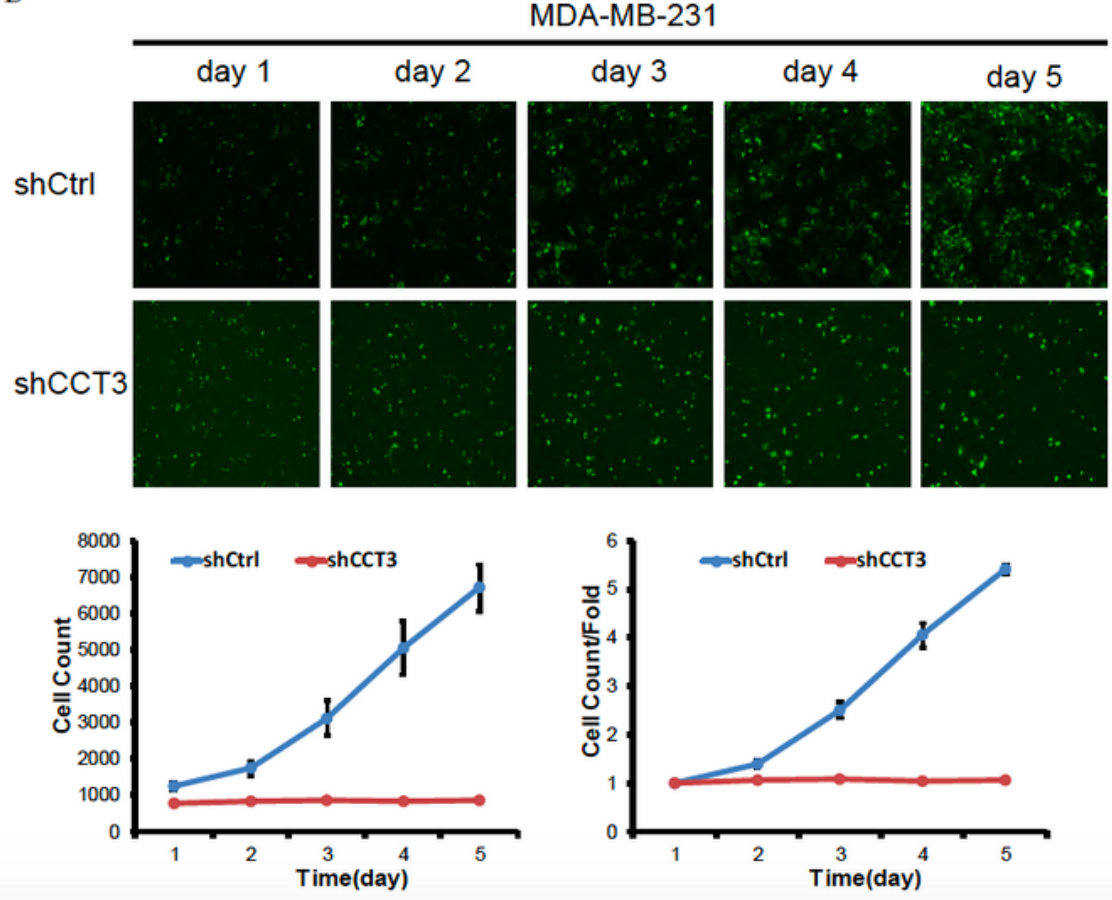

\section{Figure 2}

Knockdown of CCT3 suppresses the proliferation of breast cancer cells, HCC1937(A) and MDA-MB231(B) by celigo cytometry analysis. After infection of lentivirus shCCT3 or shCtrl, celigo imgage cytometer was used to evaluate the number of cells by scanning green fluorescence daily for 5 consecutive days and the cell proliferation curve was plotted according to the number of cells with green fluorescence. 
HCC1937
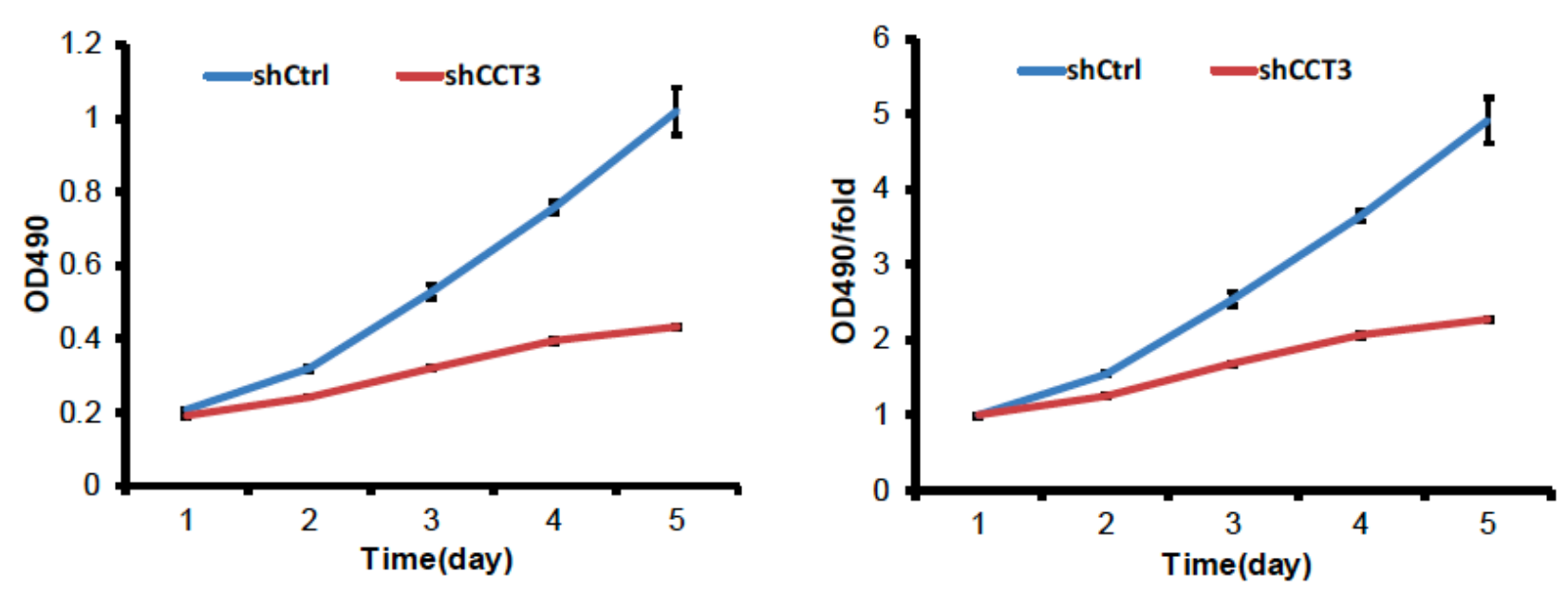

MDA-MB-231
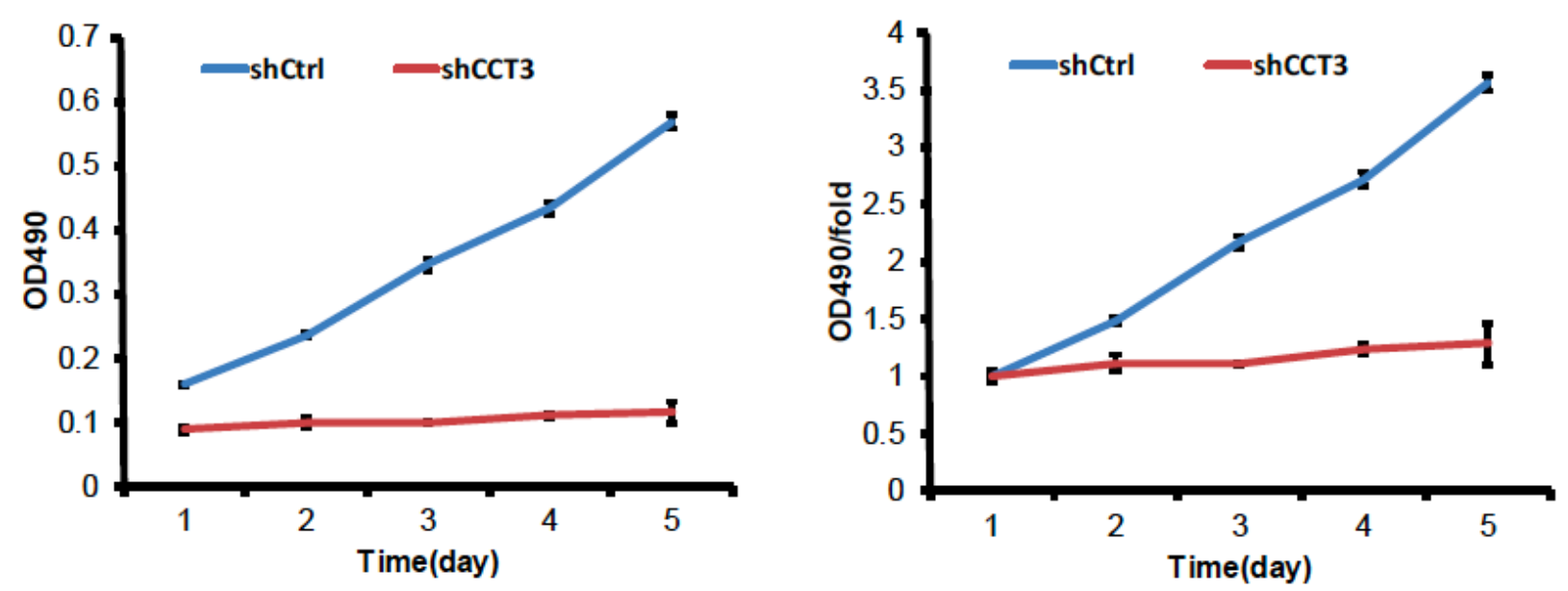

\section{Figure 3}

Knockdown of CCT3 suppresses the proliferation of breast cancer cells, HCC1937 and MDA-MB-231 by MTT assay. MTT assay was carried out at $24 \mathrm{~h}, 48 \mathrm{~h}, 72 \mathrm{~h}, 96 \mathrm{~h}$ and $120 \mathrm{~h}$ after infection of shCCT 3 and shCtrl. The number of cells was counted using a microplate reader at a wavelength of $490 \mathrm{~nm}$, and the cell proliferation curve was plotted according to the value of OD490. 
A

HCC1937

shCtrl
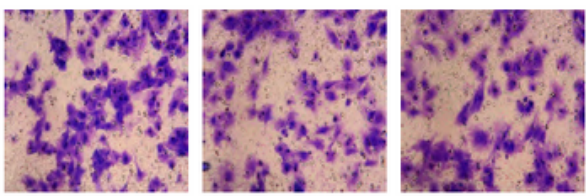

$\operatorname{shCCT3~}$
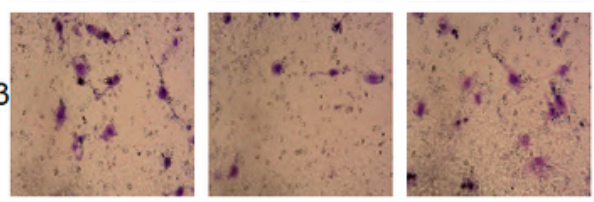

MDA-MB-231
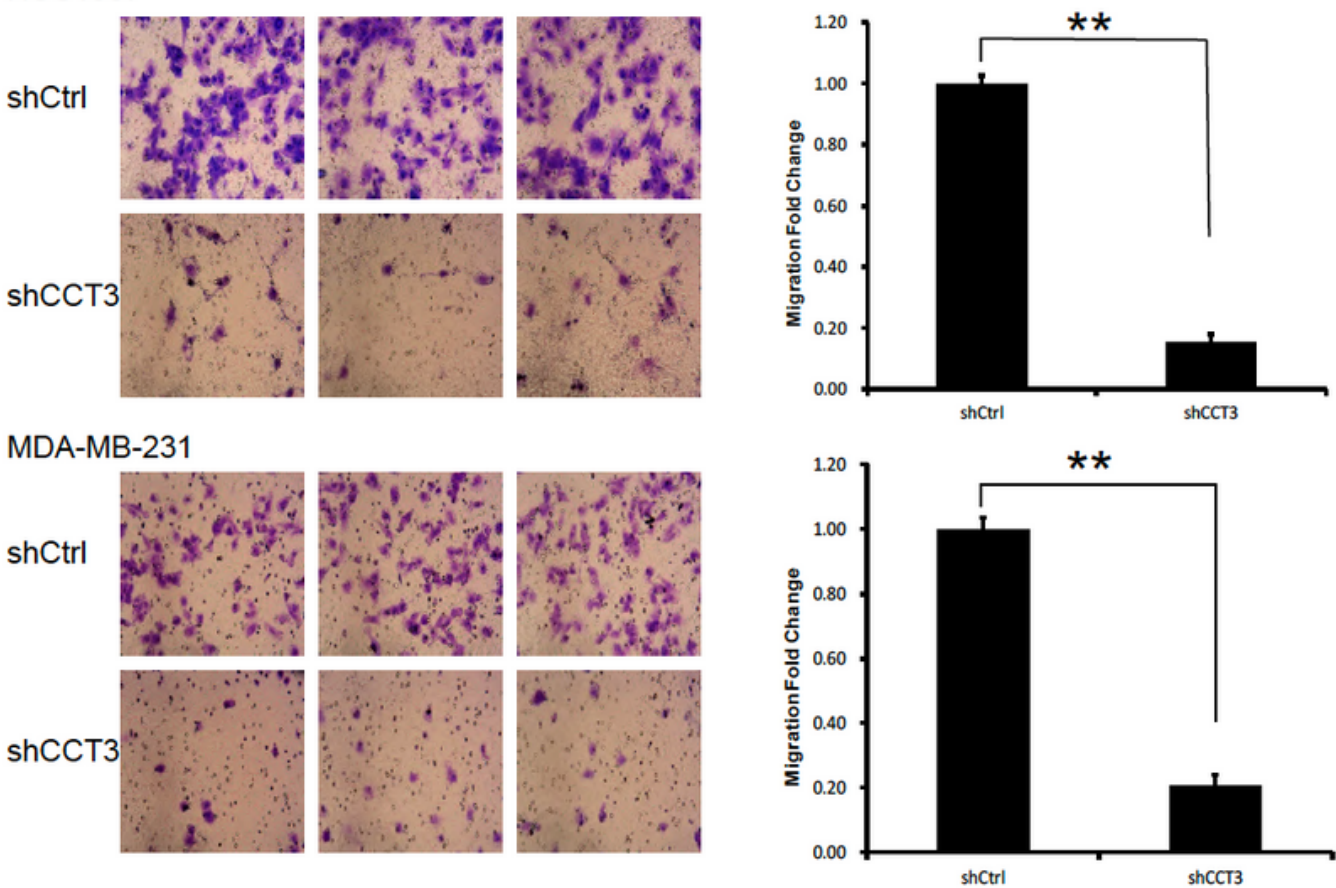

B

HCC1937

shCtrl
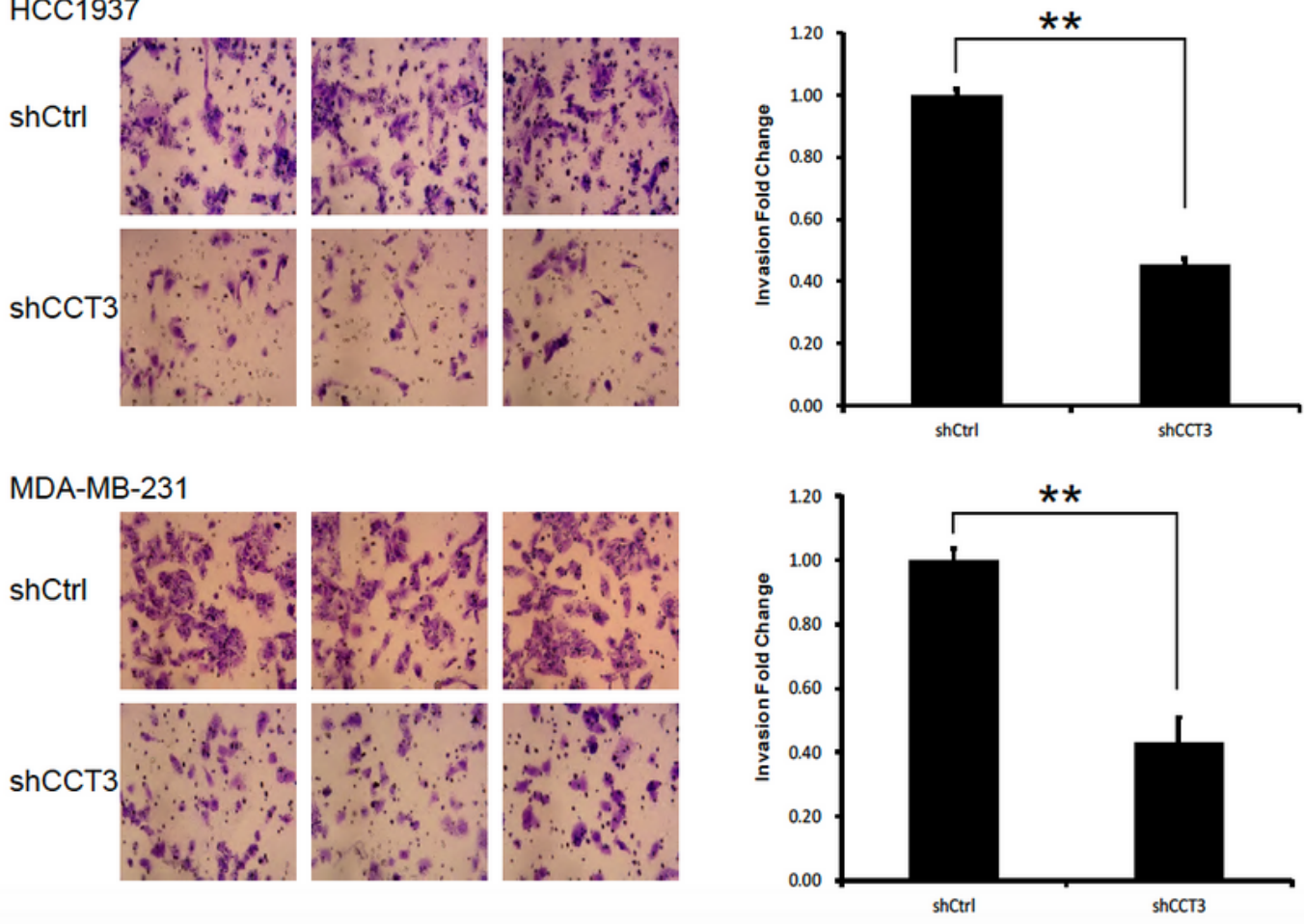

Figure 4

Knockdown of CCT3 promotes the migration of breast cancer cells. (A) Transwell assay without matrigel.

(B) Transwell assay with matrigel. The migration of breast cancer cell HCC1937 and MDA-MB-231 was analyzed by transwell assay. Original magnification $\times 200$. Data was presented as the mean \pm standard error. ** $p<0.01$ shCCT3 vs shCtrl. 
HCC1937
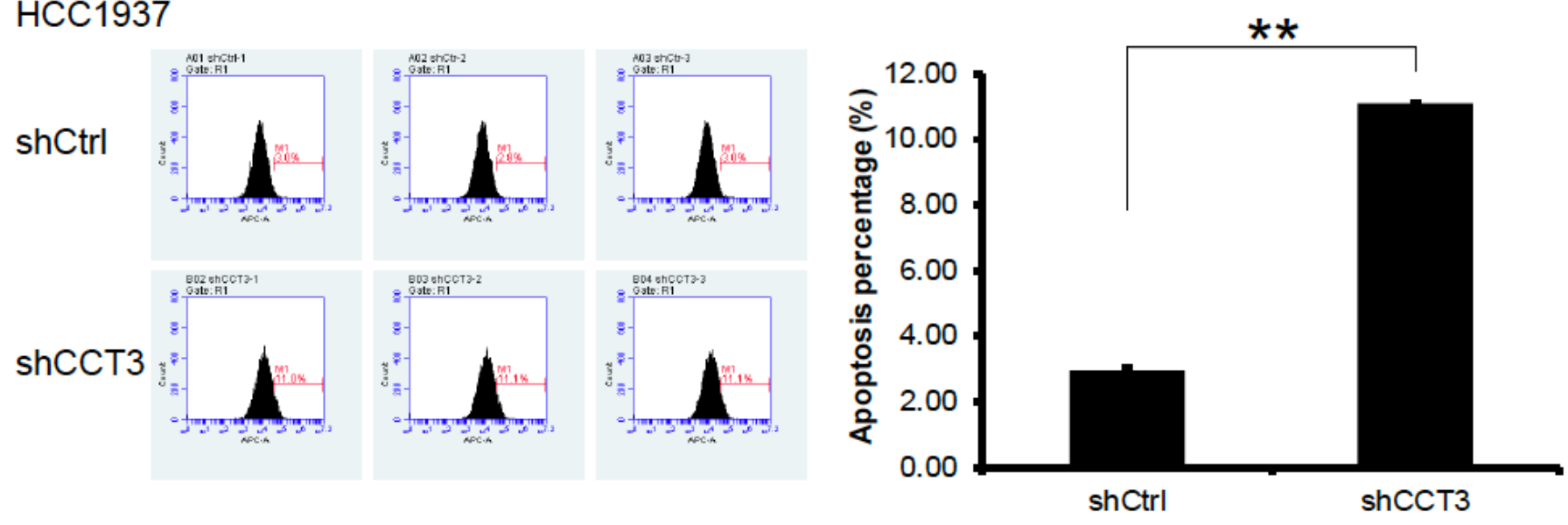

MDA-MB-231
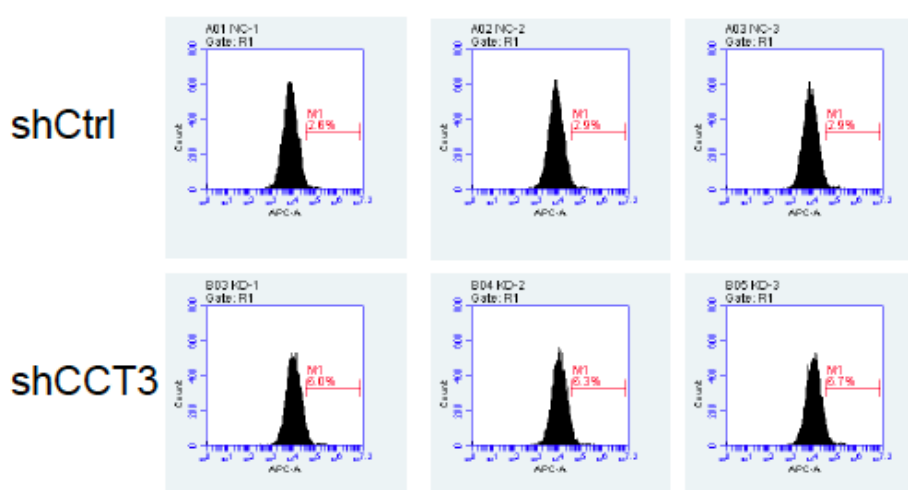

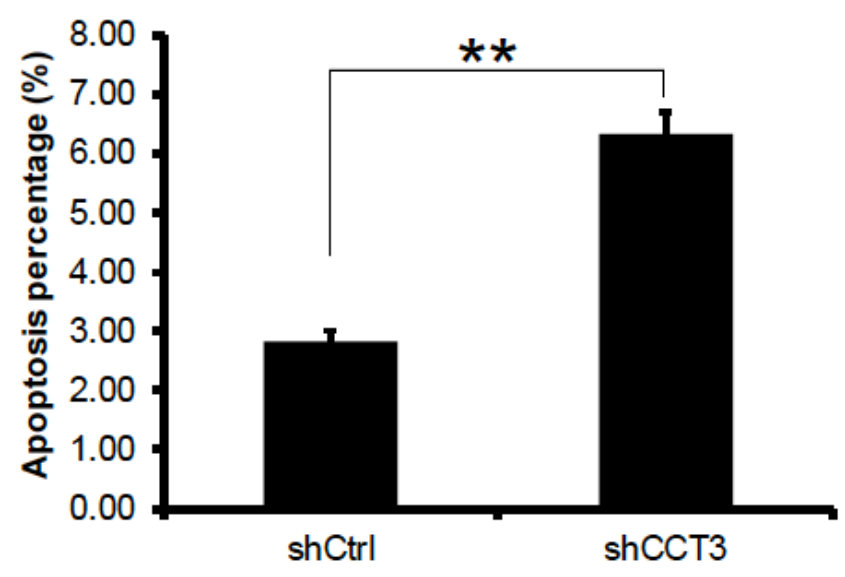

Figure 5

Knockdown of CCT3 induces the apoptosis of breast cancer cells. The apoptosis of breast cancer cell HCC1937 and MDA-MB-231 was analyzed by Annexin V-APC single staining method. Data was presented as the mean \pm standard error. ${ }^{*} \mathrm{p}<0.01$ shCCT3 vs shCtrl. 
HCC1937
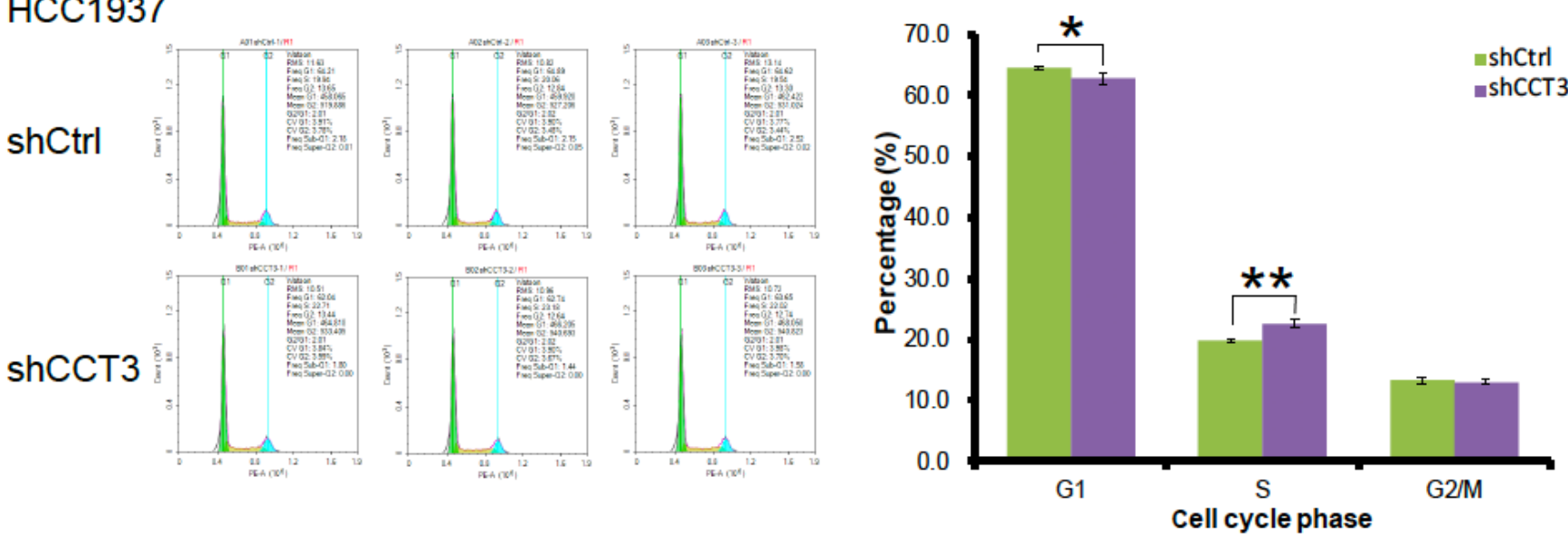

MDA-MB-231
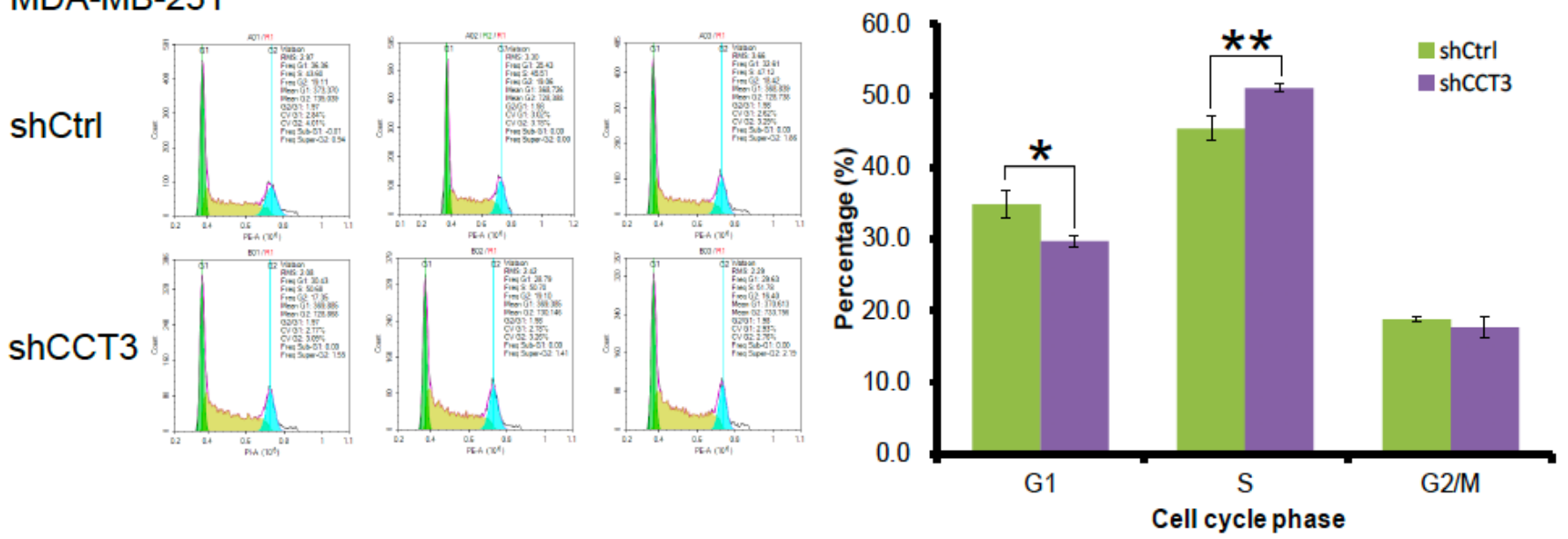

Figure 6

Knockdown of CCT3 regulates the cell cycle process in breast cancer cells. The cell cycle distribution was analyzed by flow cytometry. Data was presented as the mean \pm standard error. ${ }^{*} p<0.05,{ }^{\star} * k<0.01$ shCCT3 vs shCtrl. 


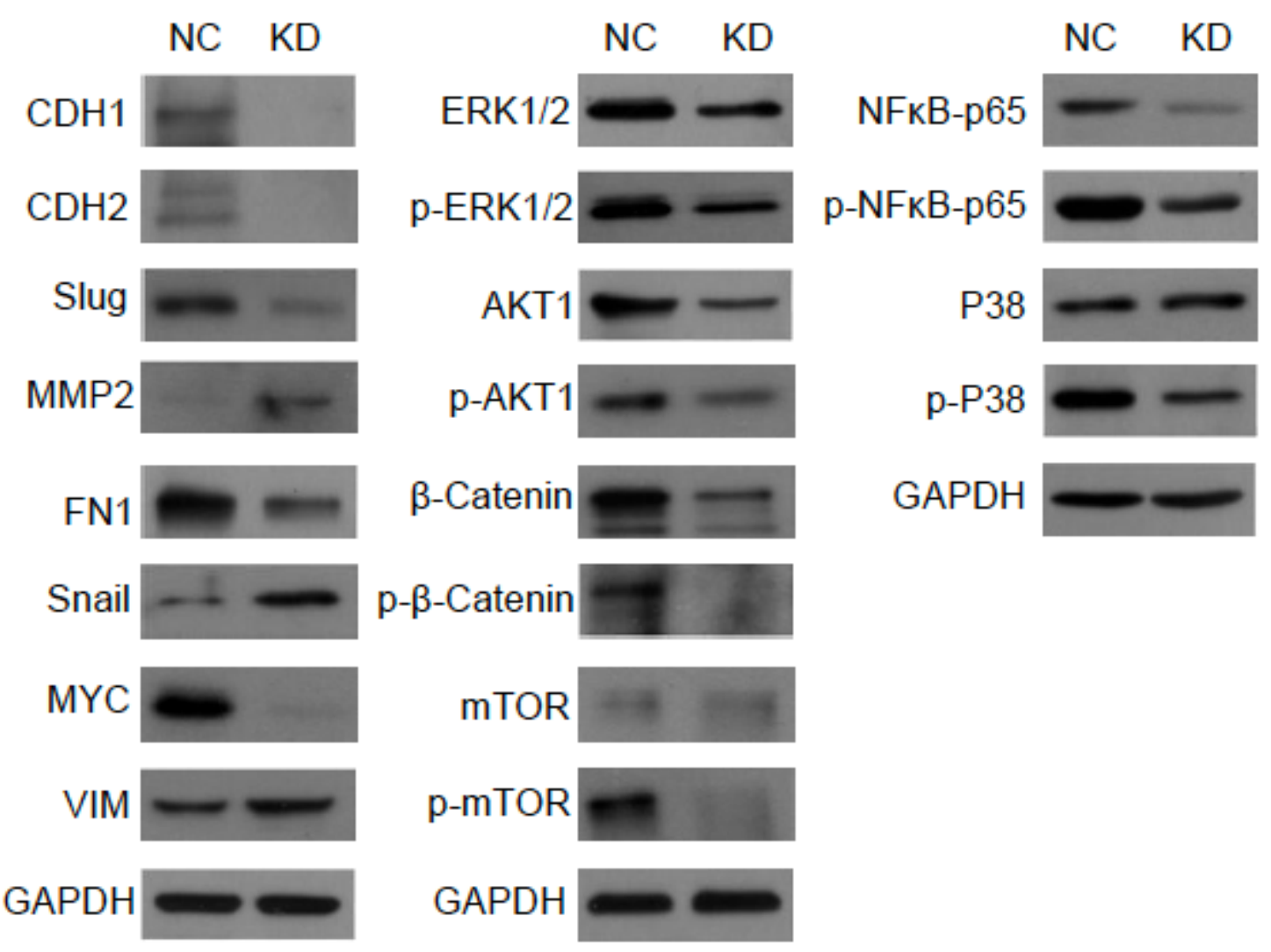

Figure 7

Knockdown of CCT3 regulates signal transduction pathway in MDA-MB-231 cells. The signal transduction protein was measured by western blot analysis, and GAPDH was used as an inner control. 